\title{
A NEW OPACITY-SAMPLING MODEL ATMOSPHERE PROGRAM FOR ARBITRARY ABUNDANCES
}

\author{
ROBERT L. KURUCZ \\ Harvard-Smithsonian Center for Astrophysics \\ 60 Garden Street, Cambridge, MA 02138, USA \\ rkuruczecfa.harvard.edu
}

\begin{abstract}
I have developed a new opacity-sampling version of my model atmosphere program called ATLAS12. It recognizes more than 1000 atomic and molecular species, each in up to 10 isotopic forms. It can treat all ions of the elements up through $\mathrm{Zn}$ and the first 5 ions of heavier elements up through Es. The elemental and isotopic abundances are treated as variables with depth. At present ATLAS12 reads atomic line data from Kurucz CDROM 1 and diatomic molecular data from Kurucz CD-ROM 15. For each line the wavelength, identification, lower energy level, gf, radiative, Stark, and van der Waals damping constants are packed into 16 bytes. At each wavelength point in a frequency integration the Voigt profiles of all the significant nearby lines are computed and summed. The $\mathrm{H}, \mathrm{He}$, and autoionizing lines are treated with the appropriate profile functions.

The fluxes predicted by ATLAS12 are not accurate in intermediate or narrow bandpass intervals because the sample size is too small. A special stripped version of the spectrum synthesis program SYNTHE is used to generate the surface flux for the converged model using the line data on CD-ROMs 1 and 15. That high resolution spectrum can be used directly or can be instrumentally broadened to compare to low resolution observations.

ATLAS12 can be used to produce improved models for Am and Ap stars. It should be very useful for investigating diffusion effects in atmospheres. It can be used to model exciting stars for H II regions with abundances consistent with those of the $\mathrm{H}$ II region. These programs and line files will be distributed on CD-ROMs.
\end{abstract}




\section{Introduction}

There are three methods for dealing with line opacity in a model stellar atmosphere. The first is direct calculation of the spectrum of all the lines which can number more than 100 million. It requires a wavelength resolution small enough to resolve the spectrum, smaller than the Doppler width. In an A star model a resolving power of 500000 produces 3.5 million points. Iterating such a model to convergence is just beyond the state of the art.

The second method is to sample the spectrum by choosing a small number of wavelength points that do not resolve the spectrum in detail. In computing a model the quantity of interest is the integral over the spectrum at each depth which determines the total energy flowing through the atmosphere. Only $1 \%$ as many points as are used in the detailed calculation are needed to get accurate integrals. This approach is called opacity sampling.

The third approach is to tabulate statistical distribution functions for the line opacity as a function of temperature and pressure for a fixed set of abundances. The complete opacity spectrum is computed for each temperature-pressure pair and then divided into small wavelength intervals. For each interval a distribution function is computed that tells the fraction of the interval having opacity less than a given value. I have performed distribution function calculations for many scaled solar abundances and I have distributed the tables on Kurucz CD-ROMs 2 to 12 . By using these tables a model can be computed for the given abundances using only a few hundred wavelength points. A large amount of computer time is required in the pretabulation but the models can be computed quickly and in large grids.

ATLAS12 is an opacity sampling program that I originally wrote in 1992 to allow computation of models with individual abundances. It is described in Kurucz (1993). The reader should consult that paper for details about ATLAS12 and about my existing grids of models computed with scaled solar abundance distribution function line opacities. That paper has figures showing sample calculations. Castelli and Kurucz (1994) present an ATLAS12 model for Vega.

ATLAS12 seemed to be working well and had no trouble producing models for A stars with abundances within a factor of three of solar. Fiorella Castelli and I were able to compute models for Vega and Sirius including variations in helium abundance. However when I subsequently tried to compute models that were $50 \%$ helium, the program would no longer converge. I tried various schemes such as computing a series of models with helium abundance gradually increasing from solar. Nothing worked. I finally understood that the program uses pretabulated Rosseland opacity in two places, in the temperature correction to account for the change in op- 
tical depth as the temperature changes, and in convection to determine the opacities of hot and cold elements. If the Rosseland table was not far off, as in the case of Vega and Sirius, the program would work because iteration made up for the small errors. But with large opacity changes, it was hopeless. The pretabulated Rosseland opacity was an end result of computing the distribution function opacities. They existed only for scaled solar abundances and each abundance required 100 Cray hours to compute. In order to make opacity sampling viable I had to find a way to determine the Rosseland opacity using no computer time. Michele Gerbaldi finally forced me to solve the problem in May 1995.

\section{Virtual Rosseland opacity}

All versions of the ATLAS model atmosphere program compute the Rosseland opacity for the temperature and pressure of each layer of the model for each iteration. ATLAS12 now saves all these values as it iterates. It finds the Rosseland opacity for convection and the temperature correction by guessing from the nearest saved values. The procedure is to divide the $\mathrm{T}, \mathrm{P}$ plane into quadrants around the point of interest, then to pick out the nearest tabulated point in each quadrant, and then to linearly interpolate among those points. Often not all the quadrants are occupied so various ad hoc guesses are made. Since these Rosseland opacities are computed for the abundances actually used in the model they can be more accurate, even when guessed, than pretabulated values. The models now converge well.

\section{Computing the whole spectrum}

When actually working with ATLAS12 sampled fluxes, Castelli and I found that, while quite accurate for predicting the total flux, the fluxes are not accurate in intermediate or narrow bandpass intervals because the sample size is too small. I wrote a special stripped version of the spectrum synthesis program SYNTHE to generate the surface flux for the converged model using the line data from CD-ROMs 1 and 15 . Plots showing sample calculations of complete spectra are given in Kurucz (1994) and Kurucz (1995). As most of the line positions are predicted, the computed flux spectrum is not realistic when compared to a high resolution observed spectrum. It is possible to select only the subset of lines with accurate wavelengths, but many lines will be missing. This difficulty can be removed only by improving laboratory spectrum analyses. When more energy levels are known, more line positions can be computed accurately.

The spectrum is computed in pieces typically at resolving power 500000 . It is actually computed as 17 intensity spectra spread across the disk of the star from center to limb. These pieces are merged into 17 continuous 
intensity spectra. Then rotationally broadened flux spectra are computed for a number of values of $\mathrm{v} \sin \mathrm{i}$, still at a resolving power of 500000, by interpolating and integrating over the disk. Alternatively, the pieces can be rotationally broadened and then merged. In these calculations all information about individual lines is thrown away. To get the complete information the calculation must be made with the full SYNTHE program. These high resolution spectra can be used directly or can be instrumentally broadened to compare to low resolution observations. The intensity spectra from different models can be combined to mimic surface features.

\section{Distribution}

I will distribute a CD-ROM with programs ATLAS12, the fast SYNTHE, a new distribution function program, and a new Rosseland opacity program. These programs all read line data from CD-ROMs 1 and 15. All the CDROMs cited in this paper can be requested from the author at the email address listed above.

\section{References}

Castelli, F. \& Kurucz, R. L. (1994) A\&A 281, 817

Kurucz, R. L. (1993) in Peculiar versus Normal Phenomena in A-type and Related Stars, M. M. Dworetsky, F. Castelli, and R. Faraggiana, eds., A.S.P. Conference Series, 87 Kurucz, R. L. (1994) in Molecules in the Stellar Environment, U. G. Jørgensen, ed., Springer-Verlag, Berlin, 282

Kurucz, R. L. (1995) in Highlights of Astronomy, Vol. 10, I. Appenzeller, ed., 407 